\title{
Intraventricular Neurocysticercosis Managed with Albendazole and Dexamethasone
}

\author{
Jan Hajek, Jay Keystone
}

Can. J. Neurol. Sci. 2009; 36: 102-104

Neurocysticercosis is the most common parasitic infection of the central nervous system and is considered the number one cause of seizures in adults worldwide ${ }^{1}$. The Worls Health Organization (WHO) estimates that neurocysticercosis affects 50 million people worldwide and causes approximately 50000 deaths annually ${ }^{2}$. However, despite its prevalence, its management remains suboptimal and controversial ${ }^{3}$. The treatment of intraventricular cysts, which occur in approximately $30 \%$ of patients with neurocysticercosis, is particularly problematic ${ }^{4}$. Mobile cysts can result in acute obstructive hydrocephalus by mechanically blocking cerebrospinal fluid (CSF) flow or by triggering an inflammatory response and ependymitis.

Intraventricular cysts may involute following cysticidal therapy with albendazole or praziquantel ${ }^{5-9}$. However, the response is often suboptimal, and they are more resistant to medical therapy than parenchymal cysts. Furthermore, by killing the parasite, cysticidal therapy itself can trigger an inflammatory response, ependymitis and hydrocephalus ${ }^{4}$. In light of these concerns and advancing technology, surgical neuroendoscopic removal has emerged as the therapeutic procedure of choice for intraventricular neurocysticercosis ${ }^{10,11}$. However, it is invasive, technically challenging, and not readily available. Furthermore, surgical removal of the cysts does not always prevent future hydrocephalus related to inflammation. There are no randomized trials comparing the usefulness and safety of medical compared with surgical approaches.

We report a case of intraventricular neurocysticercosis, complicated by acute hydrocephalus, and managed successfully with albendazole and dexamethasone.

\section{Case Report}

In October 2005, a 43-year-old female was admitted to a hospital in Toronto with acute meningoencephalitis. She had emigrated from Haiti in 2003 and, other than hypertension, was previously healthy. The day prior to admission, she developed sudden onset of headache, chills and malaise. She retired to bed, where, three hours later her husband found her very difficult to rouse and incontinent of urine. She was taken immediately to hospital. On arrival, her Glasgow Coma Scale (GCS) had improved to 15, her blood pressure was 205/107 and temperature $38.1^{\circ} \mathrm{C}$. A computed tomogram (CT) scan documented mild hydrocephalus but no evidence of intracranial space occupying lesions. Cerebrospinal fluid examination revealed $87 \mathrm{WBC} / \mu \mathrm{L}$ with $63 \%$ neutrophils. She was treated empirically with antibiotics and acyclovir and her blood pressure was controlled.
Three days later, an magnetic resonance imaging (MRI) documented resolution of the hydrocephalus and revealed cystic intraventricular lesions interpreted as possible choroid plexus cysts (Figure A). The CSF bacterial, viral, cryptococcal and mycobacterial testing were negative. Feeling better, her antimicrobials were discontinued and she was discharged on a new antihypertensive regimen.

However, within one week, she had another episode of acute headache followed by obtundation and urinary incontinence. On return to hospital, she again spontaneously regained consciousness, her blood pressure was 206/100, and temperature $38.2^{\circ} \mathrm{C}$. Cerebrospinal fluid revealed $400 \mathrm{WBC} / \mu \mathrm{L}$, now predominantly lymphocytes $(78 \%)$. Magnetic resonance imaging documented a change in the configuration of the intraventricular cysts, and a scolex characteristic of cysticercosis was visualized (Figure C). Electroencephalography was consistent with a diffuse encephalopathy with no epileptiform activity. Phenytoin was initiated prophylactically, her headache resolved, and she again regained her normal level of function. She was discharged home and referred to the Tropical Disease Unit at Toronto General Hospital.

Her neurocysticercosis serology was positive (enzyme-linked immunoelectrotransfer blot (EITB) assay CDC, Atlanta, US) and plans were made for an elective admission to hospital to initiate medical therapy.

However, before her planned admission, she again developed headache, vomiting and confusion and returned to hospital. On examination, her initial GCS was 11, but gradually normalized following a few hours of observation. There was no papilledema. The MRI documented generalized ventricular enlargement and revealed that the cysts had changed in appearance. The cyst in the fourth ventricle had grown in size, acquired proteinaceous material and moved towards, possibly blocking, the cerebral aqueduct (Figure E). There was no enhancement or neuroimaging evidence of ependimytis. She was admitted to the neurosurgery intensive care unit for close observation and

From the Division of Infectious Diseases, North York General Hospital (JH); Tropical Disease Unit, Division of Infectious Diseases, The Toronto Hospital (JK), University of Toronto, Toronto, Ontario, Canada.

Received August 17, 2008. Final Revisions Submitted SePtember 15, 2008. Correspondence to: Jan Hajek, Department of Infectious Diseases, North York General Hospital, 4001 Leslie Street, GW-33, Toronto, Ontario, M2K 1E1, Canada. 


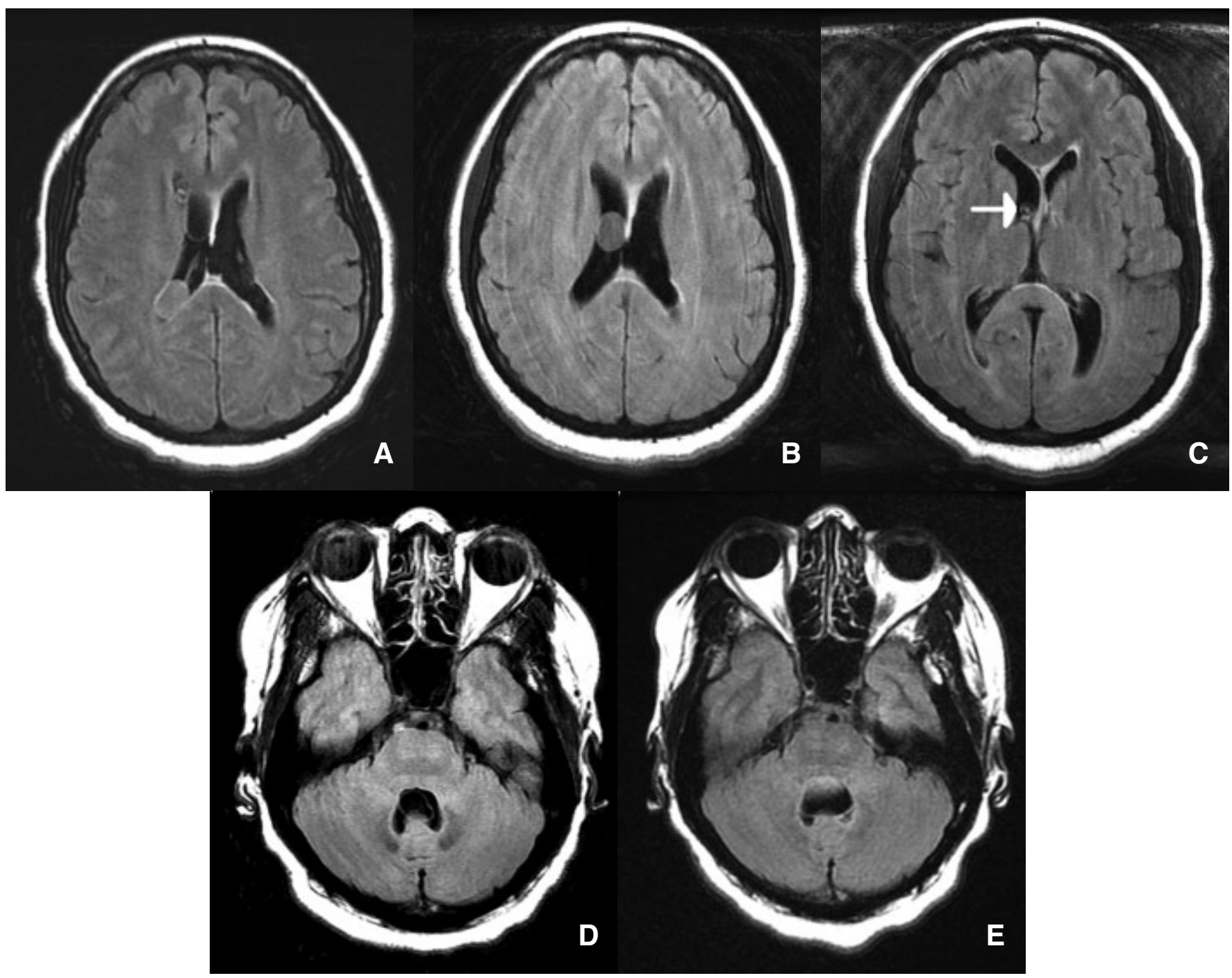

Figure: Axial FLAIR MR images of a 43 year old patient with neurocysticercosis. The configuration and appearance of intraventricular cysts in the lateral ventricles changed from October $31(A)$ to November $7(B)$ and a pathogonomic scolex was identifiable in the lateral ventricle in November $(C)$. Between November $(D)$ and January $(E)$ an intraventricular cyst in the 4th ventricle increased in size and density and began to obstruct the ventricle resulting in an episode of acute hydrocephalus. See text for details.

medical therapy with dexamethasone, $16 \mathrm{mg}$ per day, and albendazole, $15 \mathrm{mg} / \mathrm{kg}$ per day was initiated. Over the next week, she showed continued improvement, she became alert and her headache resolved. Clinically stable and asymptomatic she was discharged from hospital to complete a 30-day course of albendazole. On repeat MRI four months later there was no residual hydrocephalus, no evidence of intraventricular cysts, just a single focal area of parenchymal calcification, unchanged from all her previous studies. Over six months, her steroids were tapered slowly and intermittently because of recurrence of headache; phenytoin was discontinued. Other than occasional headaches, she remains well two years following therapy.

\section{Discussion}

Intraventricular neurocysticercosis is a challenging clinical problem. With an attendant risk of precipitating acute hydrocephalus or ependymitis, it carries a worse prognosis and is more difficult to treat than parenchymal disease. Even diagnosis is difficult as viable intraventricular cysts are often not seen on CT scan

We describe a patient with intraventricular neurocysticercosis who presented with episodes of acute intermittent hydrocephalus. This likely resulted from mechanical obstruction of CSF flow and an inflammatory response as the cysts began to die as evidenced by changes in their MRI appearance and the raised CSF WBC count. She demonstrated clinical response and 
radiological cure following a 30-day course of albendazole (15 $\mathrm{mg} / \mathrm{kg} /$ day) and a six-month course of steroids.

The utility and safety of medical therapy in intraventricular neurocysticercosis is not clear ${ }^{3}$. Recent randomized trials and a meta-analysis, have established that cysticidal therapy in conjunction with steroids is beneficial in treatment of parenchymal neurocysticercosis both in achieving radiological cure and reducing seizure recurrence ${ }^{12}$. However, intraventricular cysts are more recalcitrant to medical therapy and frequently require surgical intervention to manage associated hydrocephalus. In a recent trial of extraparenchymal neurocystisercosis only 6 of 14 patients treated with albendazole $15-30 \mathrm{mg} / \mathrm{kg}$ for eight days had $\geq 50 \%$ reduction in cyst size at six months ${ }^{8}$. Many were also treated with ventriculoperitoneal (VP) shunting prior to therapy ${ }^{8}$. Proaño et al reported more favourable results ${ }^{9}$. Following a two week course of albendazole $(15 \mathrm{mg} / \mathrm{kg} /$ day $)$, eight of ten patients had $\geq 80 \%$ decrease in size of intraventricular cysts ${ }^{9}$. With additional courses of albendazole these eight patients had complete radiological resolution and did not require surgical intervention ${ }^{9}$. Although shorter courses of albendazole are effective for parenchymal cysts, because of the relative resistance of intraventricular cysts we decided to prolong albendazole to 30 days $^{13}$.

Surgical removal promises a fast and definitive method of removing the cysts and eliminating the risk of acute mechanical obstruction $^{10.11}$. However, even following effective cyst removal, chronic hydrocephalus, related to inflammatory damage, can still occur. Ventriculostomy, followed by insertion of a ventriculoperitoneal shunt, is often necessary for manage-ment of hydrocephalus. However, VP shunting in the setting of neurocystisercosis has frequent complications, particularly obstruction, requiring frequent revisions and carrying a high attendant mortality ${ }^{14}$.

Our patient presented with evidence of acute intermittent hydrocephalus likely due to mechanical obstruction. Following her last admission we strongly considered surgical intervention. However, our centre's experience with neuroendoscopy, particularly in the treatment of neurocysticercosis, is limited. As she had rapid improvement of her level of consciousness after admission, we held off on surgical intervention and initiated medical therapy. She was admitted to the neurosurgical intensive care unit and closely observed with a contingency plan of urgent ventricular shunting should she clinically deteriorate.

This case report adds to the medical literature demonstrating that albendazole and dexamethasone can be effective in management of symptomatic intraventricular neurocysticercosis. In this case, it may have obviated the need for ventricular shunt placement and surgical procedures in the posterior fossa. However, the destruction of the cysts in the ventricles with antiparasitic therapy may increase the host inflammatory response and the risk of ependymitis. Despite the use of steroids during antiparasitic therapy, chronic hydrocephalus can still develop years later requiring VP shunting ${ }^{4}$. These shunts are at high risk of obstruction, and some authors have advocated prolonged courses of steroids to prevent this complication ${ }^{15}$. Prior to treatment, our patient had evidence of ependymitis manifest by CSF leukocytosis. Because of her recurrent headaches there was an apparent need to continue corticosteroid therapy for six months. This re-emphasizes the differences in medical therapy of intraventricular neurocysticercosis compared to the more common parenchymal forms of infection ${ }^{4}$.

Both medical and surgical therapy likely play a role in eradicating the parasite and minimizing the inflammatory response. Given the heterogeneity of presentations, the lack of randomized controlled trials, and the potentially devastating complications, the management of intraventricular neurocysticercosis can be extremely challenging.

\section{REFERENCES}

1. Carpio A. Neurocysticercosis: an update. Lancet Infect Dis. 2002;2: 751-62.

2. Schantz PM, Cruz M, Sarti E, Pawlowski Z. Potential eradicability of taeniasis and cysticercosis. Bull Pan Am Health Organ. 1993; 27(4):397-403.

3. Nash TE, Singh G, White AC, Rajshekhar V, Loeb JA, Proano JV, et al. Treatment of neurocysticercosis: current status and future research needs. Neurology. 2006 Oct 10;67(7):1120-7.

4. Cuetter AC, Andrews RJ. Intraventricular neurocysticercosis: a series of 18 consecutive patients and review of the literature. Neurosurg Focus. 2002;12(6):e5.

5. Allcut DA, Coulthard A. Neurocysticercosis: regression of a fourth ventricular cyst with praziquantel. J Neurol Neurosurg Psychiatry. 1991 May;54(5):461-2.

6. Fandino J, Botana C, Fandino C, Rodriguez D, Gomez-Bueno J. Clinical and radiographic response of fourth ventricle cysticercosis to praziquantel therapy. Acta Neurochir. 1991; 111(3-4):135-7.

7. del Brutto $\mathrm{OH}$, Sotelo J. Albendazole therapy for subarachnoid and ventricular cysticercosis. Case report. J Neurosurg. 1990 May; 72(5):816-7.

8. Gongora-Rivera F, Soto-Hernandez JL, Gonzalez Esquivel D, Cook HJ, Marquez-Caraveo C, Hernandez Davila R, et al. Albendazole trial at 15 or $30 \mathrm{mg} / \mathrm{kg} /$ day for subarachnoid and intraventricular cysticercosis. Neurology. 2006 Feb 14;66(3):436-8.

9. Proaño JV, Madrazo I, García L, García-Torres E, Correa D. Albendazole and praziquantel treatment in neurocysticercosis of the fourth ventricle. J Neurosurg. 1997;87:29-33.

10. Torres-Corzo J, Rodriguez-della Vecchia R, Rangel-Castilla L. Bruns syndrome caused by intraventricular neurocysticercosis treated using flexible endoscopy. J Neurosurg. 2006 May;104(5):746-8.

11. Garcia HH, Evans CA, Nash TE, Takayanagui OM, White AC Jr, Botero D, et al. Current consensus guidelines for treatment of neurocysticercosis. Clin Microbiol Rev. 2002 Oct;15(4):747-56.

12. del Brutto OH, Roos KL, Coffey CS, García HH. Meta-analysis: Cysticidal drugs for neurocysticercosis: albendazole and praziquantel. Ann Intern Med. 2006 Jul 4;145(1):43-51.

13. Sotelo J, del Brutto OH, Penagos P, Escobedo F, Torres B, Rodriguez-Carbajal J, et al. Comparison of therapeutic regimen of anticysticercal drugs for parenchymal brain cysticercosis. J. Neurol. 1990;237:69-72.

14. Colli BO, Carlotti CG Jr, Assirati JA Jr, Machado HR, Valenca M, Amato MC. Surgical treatment of cerebral cysticercosis: longterm results and prognostic factors. Neurosurg Focus. 2002 Jun $15 ; 12(6): \mathrm{e} 3$.

15. Suastegui Roman RA, Soto-Hernández JL, Sotelo J. Effects of prednisone on ventriculoperitoneal shunt function in hydrocephalus secondary to cysticercosis: a preliminary study. J Neurosurg. 1996;84:629-33. 